\title{
A Pedestrian Navigation System using Navigation Sentence based on XML
}

\author{
Zheng Pan $^{1,3}$ Lei Yan ${ }^{1}$ Adam C. Winstanley ${ }^{2}$ A.Stewart Fotheringham ${ }^{3}$ Jianghua Zheng ${ }^{2}$ \\ 1. Beijing Key Lab of Spatial Information Integration \& Its Applications, Institute of RS\&GIS, Peking University, Beijing, China \\ 2. Department of Computer Science, National University of Ireland, Maynooth, Co. Kildare, Ireland \\ 3. National Centre for Geocomputation, National University of Ireland, Maynooth, Co. Kildare, Ireland
}

\begin{abstract}
Pedestrian navigation system has been widely used in many fields. In order to offer navigation information intelligible for pedestrian, we provide navigation sentences to users, which can be easily displayed on cellular phones with small display area. In addition, the navigation sentences can be converted into voice, which is useful for users in some special situations or with visually impairment. Navigation sentences can separately help pedestrians to go to the destination, or can be combined with electronic map to provide more intuitive navigation information. Compared with guidance maps, navigation sentences are easy to transmit and manage. The navigation sentences are described by XML (eXtensible Markup Language) and can be created on the server automatically based on the users' position and destination. This paper will discuss the structure of the pedestrian navigation system using navigation sentences. The structure and generating process of navigation sentences are also described.
\end{abstract}

Keywords-Pedestrian navigation system; XML; Text-to-speech

\section{INTRODUCTION}

It is not always easy for pedestrians to find the right way to reach their destination in an unfamiliar environment. Pedestrian navigation systems can help them to find an optimal route. According to the development of mobile phones, positioning technologies and wireless networking infrastructures, pedestrian navigation systems have become an active research field in recent years.

Different from car navigation system, the user of pedestrian navigation system should have a small client, such as cellular phone, PDA. In addition, the cellular phone is more and more miniaturized. It has a small display area, and cannot present a lot of information [1].

There are some pedestrian navigation systems available, but most of them only provide navigation data based on maps. These systems need to transmit and manage a lot of data. Thus, they can only be used on clients with high performance and big display area, which limits the further use of pedestrian navigation system [2].

To solve the problem, several studies have been made on presentation method. Textual navigation information is a kind of solution, which is easy to display even on a very small screen. As a presentation method for a small display, a study carried out route-guide map adjustment, information supplement with automatically creating navigation sentences [3] [4].

In this paper, we propose a solution with textual navigation information, called "navigation sentence". In addition, with the speech engine, such as Microsoft Text-to-speech engine, navigation sentence can be easily converted into speech, which can be used to navigate for these people with visually impairment.

In this paper, we will focus on the task of how to create navigation sentences on the server automatically. Using proposed method, our system can provide the route guide using navigation sentences, which is available for very small display of a cellular phone and for pedestrians. In section 2, we will describe the navigation information and the structure of navigation sentence. In section 3, the system structure will be given. Finally, section 4 will present conclusions and the directions that we will be taking as we continue to develop the system.

\section{NAVIGATION SENTENCE}

Compared with car navigation system, pedestrian navigation system needs more detail information. In order to provide specific navigation information, we have added many detailed information in our system.

\section{A. Navigation Information}

Navigation sentences can describe the path from start point of users to the destination. For example, "Please face northeast" and "turn right, follow the path, about 23 meters". Thus it is easy for user to grasp the relation between the place which you are and this navigation point.

A calculated path is consisted of a series of points. The process of navigation is guiding pedestrian from 
the current point to next point. In some pedestrian navigation systems, points have two attributes at most: position and name. It is easy to show the position of a point on maps, but it is not easy to describe the path clear to users only with two attributes of points. In our system, we define five attributes for each point: position, direction, movement, name and distance.

\section{Position}

After getting the users' original point and destination, we can calculate the path based on the pedestrian path planning algorithm [5]. At the same time, we can also get the longitude and latitude of each turning point on the path.

\section{Direction}

We used to tell other people "turn right at the next corner" or "turn left and go straight on". But in some cases, it is not very clear if we say turn right or left, especially when there are several forks in the road, as shown in Fig. 1.
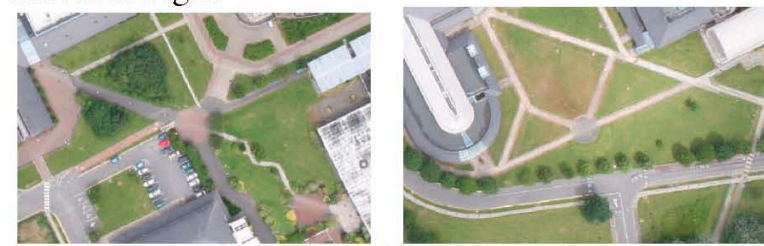

Fig. 1. Examples of sidewalk

To provide more specific information to pedestrians, we define seven kinds of directions: go straight, turn bear right, turn right, turn sharp right, turn bear left, turn left and turn sharp left, as shown in Fig.2.

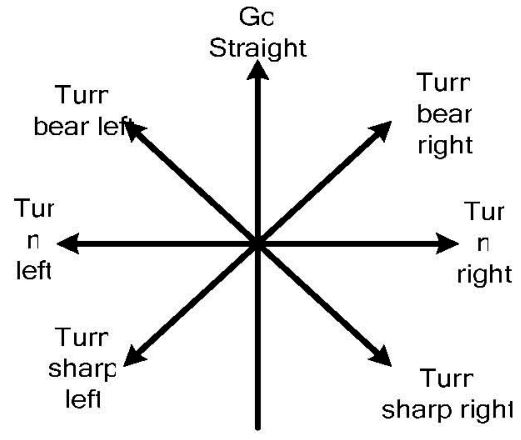

Fig. 2. Turning directions

The direction attribute is determined by the relation between current point and the next point on the calculated path.

\section{Movement}

We have defined four kinds of movement: go straight, follow, go upstairs, and go downstairs. The movement attribute is also determined by the relation between current point and the next point. We use Att_M to represent the movement attribute, the process of determining the value of Att_M is shown in Fig.3.

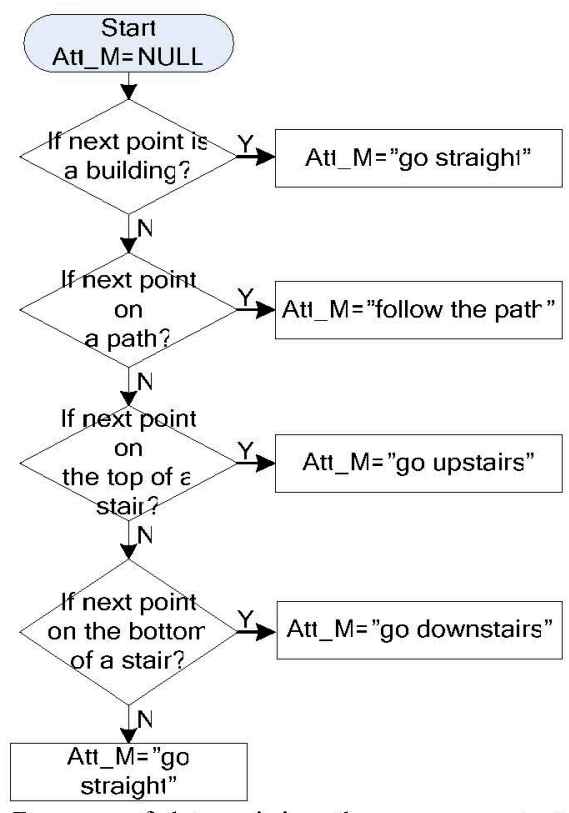

Fig.3. Process of determining the movement attribute Name

We give each point a name. It might be the name of a building, or name of a street corner, or name of a road. In particular, if the point represents a door of some building, we take the type of the door as its name, such as, sliding door or automatic door. As a result, users can receive navigation sentence like "enter John Hume building by automatic door".

\section{Distance}

The distance attribute is the distance from the current point to the next point. It can be calculated by the coordinates of current point and the next point.

\section{B. The Structure of Navigation Data}

To navigate pedestrians from a station to a destination, we define navigation data in XML. The reasons why XML attracts an attention are given below.

- XML is a universal format. XML dose not depend on a hard ware, OS, and application programs. XML only depends on a character code.

- XML data can be processed in a computer strictly and be read by a man. XML data can be processed according the name of a factor and contents, an attribute and the value. Additionally, a man can read the data and edit with a general editer, because it consists of a character string.

- XML is a general-purpose format. XML is a Meta language and dose not limits the application field. The structure of navigation data appears in Fig.4. The structure can be classified into two main groups, head and body. The head consists of title, version, date, the information about the start point and end point. The 
body consists of the information about turning direction, navigation data appears in Fig.5. point name, text guidance and distance. The example of

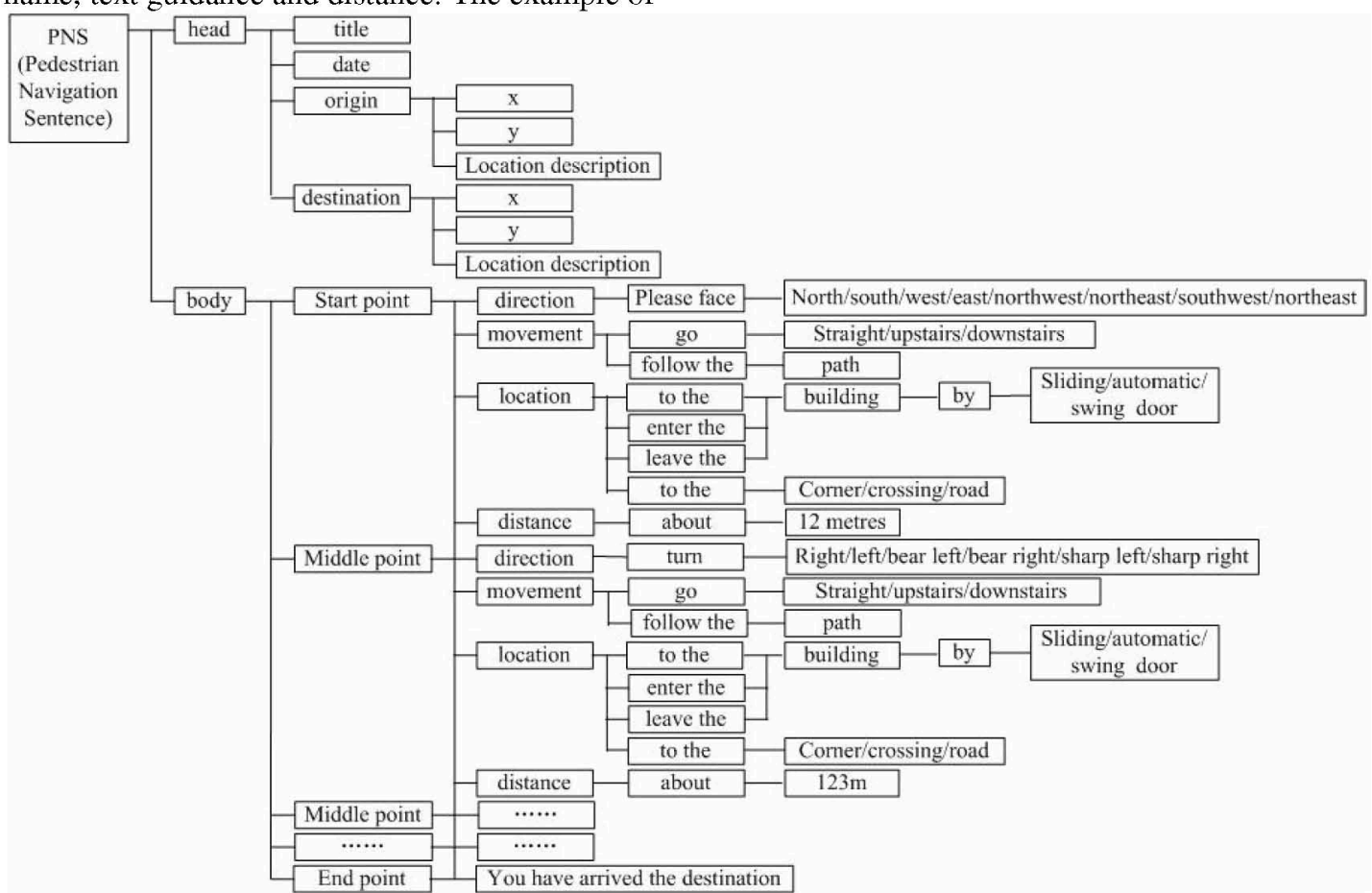

Fig.4. Structure of navigation data

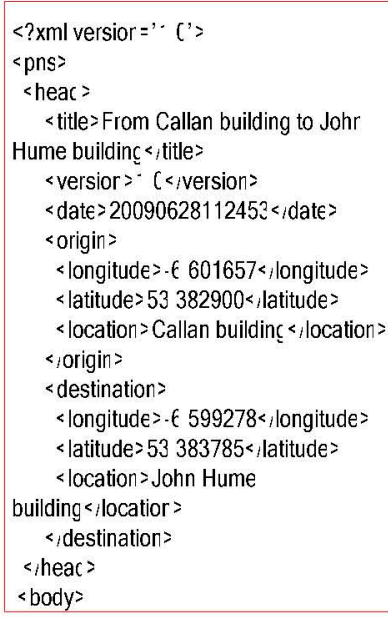

As shown in Fig.4, the structure of body is classified into three kinds of points. Except start point and end point, the other points are grouped to middle point.

Start point and middle point are different in direction attribute. At start point, pedestrians need to know the initial direction before they take their first step. When pedestrians reach one of the middle points, they only need to know turn right or left. Therefore, we define the direction attribute of start point as "please

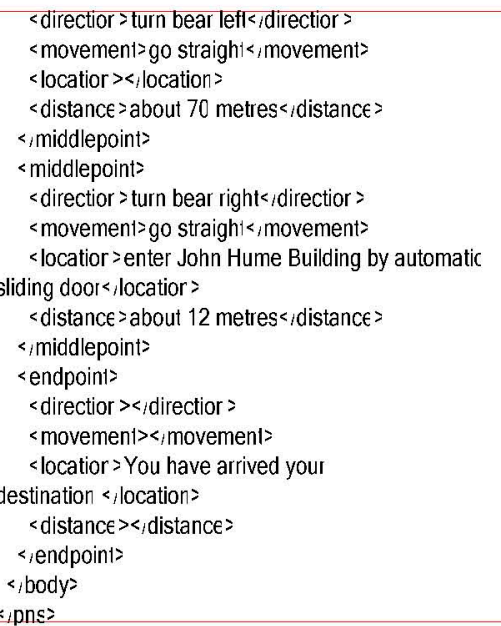

pns>

Fig.5. An example of navigation data

face north/south/..." And the direction attribute of middle points are defined as "turn right/left/..."

\section{SYSTEM DESIGN}

This section describes the design of our system. This system can plan three kinds of route for pedestrian: shortest length path, easy-walk priority path and indoor priority path [5]. In addition, there are two modes of pedestrian route description: textual mode and voice mode. Comparing with the car navigation system, 
pedestrian navigation system can provide more details and more specific information.

This system is based on Client/Server structure, as shown in Fig. 6.

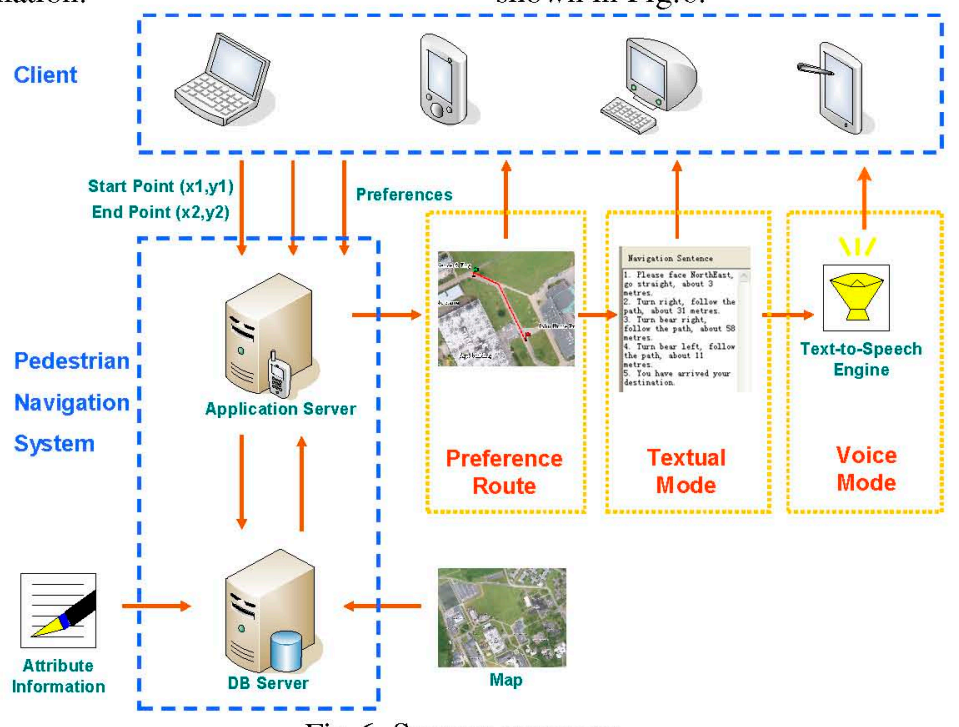

Fig.6. System structure

The process of creating navigation sentences is as follows:

- Pedestrian sends his current position and destination to application server.

- At the same time, pedestrian decides which kind of route he wants to take and sends it to application server too.

- Application server gets map information from database server.

- Application server calculates path based on pedestrian way finding algorithm.

- A route from original point to destination is calculated based on user preferences.

- Application server gets attributes information from database server.

- With detail attributes information, calculated path is translated into walking instructions given in terms of paths, landmarks and points of interest, which is easy to display and can be used without map.

- Textual route is converted into spoken voice by Microsoft Text-to-Speech Engine, which is easy to understand and suitable for blind people.

\section{CONCLUSION}

This paper has described a pedestrian navigation system using navigation sentences. These sentences are described by XML (eXtensible Markup Language). Our proposal has the following features. At first, in order to offer navigation information intelligible for a user, more details is integrated in the database and can provide more specific information to users. We provide a navigation sentence at each key point or turning point.
Secondly, navigation sentences in textual mode are easy to store and distribute. The navigation sentence is created by the application server automatically and is sent to users through Internet. Proposed system can realize intelligible way guidance for users, and provide the route guide, which are available for a small display area of a cellular phone and for pedestrians.

\section{REFERENCES}

[1] Maiko Moro, Kenichiro Tanaka, Yuka Utagawa, Hiroshi Shigeno, Yutaka Matsushita. A Pedestrian Navigation System using XML based Data. Proceedings of SPIE, Vol. 4529, 2001 SPIE

[2] Dominique Bonte. The Mobile World Congress 2008: Pedestrian Navigation at Last, 11 Feb. 2008.

[3] K. Fujii, K. Sugiyama. Route Guidance for Human Navigation Assist. International Conference on Cognitive Science, 1999.

[4] K. Fujii, K. Sugiyama. A Method of Generating a Spot-Guidance for Human Navigation. IEICE Transactions, Vol. J82-D-11, No. 11, pp. 2026-2034, Nov. 1999.

[5] Zheng Pan, Lei Yan, Adam C. Winstanley, A.Stewart Fotheringham, Jianghua Zheng. A 2-D ESPO algorithm and its application in pedestrian path planning considering human behaviour. Proceedings of the $3^{\text {rd }}$ International Conference on Multimedia and Ubiquitous Engineering (MUE 2009) 